\title{
NGC 2580 and NGC 2588
}

\section{Two open clusters in the Third Galactic Quadrant ${ }^{\star, \star \star}$}

\author{
G. Baume ${ }^{1,2}$, A. Moitinho ${ }^{3}$, E. E. Giorgi ${ }^{2}$, G. Carraro ${ }^{1}$, , and R. A. Vázquez ${ }^{2}$ \\ 1 Dipartimento di Astronomia, Università di Padova, Vicolo Osservatorio 2, 35122 Padova, Italy \\ 2 Facultad de Ciencias Astronómicas y Geofísicas de la UNLP, IALP-CONICET, Paseo del Bosque s/n, La Plata, Argentina \\ 3 CAAUL, Observatório Astronómico de Lisboa, Tapada da Ajuda, 1349-018 Lisboa, Portugal
}

Received 2 October 2003 / Accepted 21 December 2003

\begin{abstract}
We present CCD broad band photometric observations in the fields of the Third Galactic Quadrant open clusters NGC 2580 and NGC $2588\left(V(I)_{C}\right.$ and $U B V(R I)_{C}$ respectively). From the analysis of our data we found that NGC 2580 is located at a distance of about $4 \mathrm{kpc}$ and its age is close to $160 \mathrm{Myr}$. As for NGC 2588, it is placed at about $5 \mathrm{kpc}$ from the Sun and is 450 Myr old. This means that NGC 2588 belongs to the extension of the Perseus arm, whereas NGC 2580 is closer to the local arm structure. The luminosity functions (LFs) have been constructed for both clusters down to $V \sim 20$ together with their initial mass functions (IMFs) for stars with masses above $M \sim 1-1.5 M_{\odot}$. The IMF slopes for the most massive bins yielded values of $x \approx 1.3$ for NGC 2580 and $x \approx 2$ for NGC 2588. In the case of this latter cluster we found evidence of a core-corona structure produced probably by dynamical effect. In the main sequences of both clusters we detected gaps, which we suggest could be real features.
\end{abstract}

Key words. Galaxy: open clusters and associations: individual: NGC 2580, NGC 2588 - Galaxy: open clusters and associations: general

\section{Introduction}

This study is part of a long term project based on broad band CCD photometric observations of open clusters, aimed at investigating the spiral structure and the star formation history in the third Galactic quadrant; it is of particular interest to recognise the shape of the Vela-Puppis region (Moitinho 2001, 2002; Giorgi et al. 2002).

Open clusters are excellent targets to be used as natural laboratories in order to understand several issues related to the structure, the chemical population, the dynamical evolution and the stellar formation processes in the Galaxy. In this study, we pay attention to the open clusters NGC 2580 and NGC 2588. Both objects have been poorly studied up to now (identification and eye estimates of their angular size and richness). Preliminary results of NGC 2588 using part of the present data were given earlier by Moitinho (2001, 2002).

Both clusters are situated in a zone of the Galaxy where, according to Neckel \& Klare (1980), the visual absorption rises

Send offprint requests to: G. Baume, e-mail: baume@pd.astro.it

* Based on observations $\mathrm{c}$ ollected at ESO, CASLEO and CTIO.

$\star \star$ Table 3 is only available in electronic form at the CDS via anonymous ftp to cdsarc.u-strasbg.fr $(130.79 .128 .5)$ or via http://cdsweb.u-strasbg.fr/cgi-bin/qcat?]/A+A/417/961 monotonically up to about $1 \mathrm{kpc}$ from the Sun and is then nearly constant with a value $\sim 0.5^{\mathrm{m}}$ up to a distance of $2 \mathrm{kpc}$. At this distance, the absorption makes a significant jump reaching $2^{\mathrm{m}}$, a value that apparently persists up to as far as 5 or $6 \mathrm{kpc}$. The coordinates of the clusters taken from Dias et al. (2002) are shown in Table 1.

In this paper we present the first estimate of the clusters' fundamental parameters: distance, reddening, size and age, as well as their Luminosity and Initial Mass Functions. All these parameters will provide us with valuable clues for clarifying the issues enumerated above. For this purpose, $V(I)_{C}$ observations in the direction of NGC 2580 and $U B V(R I)_{C}$ in the case of NGC 2588 were performed. These optical measurements were complemented with infrared data taken from the 2MASS catalogue and with astrometric information available in the Tycho-2 catalogue (Høg et al. 2000) for the brightest cluster stars.

The plan of this study is as follows: in Sect. 2 we briefly present the observations and data reduction procedure. In Sect. 3 we describe the methods used in the determination of the basic cluster parameters. In Sect. 4 we present our results for NGC 2580 and NGC 2588. Section 5 is dedicated to a discussion and, in Sect. 6, we end with some remarks. 
Table 1. Central coordinates of the observed objects.

\begin{tabular}{ccccc}
\hline \hline Name & $\alpha_{2000}$ & $\delta_{2000}$ & $l$ & $b$ \\
\hline NGC 2580 & $08: 21: 28.0$ & $-30: 18: 00.0$ & $249.90^{\circ}$ & $+3.69^{\circ}$ \\
NGC 2588 & $08: 23: 10.0$ & $-32: 58: 30.0$ & $252.29^{\circ}$ & $+2.45^{\circ}$ \\
\hline
\end{tabular}

\section{Data set}

\subsection{Observations}

Our data set comes from CCD photometric observations carried out in the course of several runs on different telescopes:

1. The European Southern Observatory (ESO): CCD VI observations of the fields of NGC 2580 and NGC 2588 using the new EMMI read arm camera at the NTT in the photometric night of December 9, 2002 with seeing values near to $1^{\prime \prime}$. This new camera has a mosaic of two $2048 \times 4096$ pixels CCDs which samples a 9!9 × 9! 1 field. The images were binned $2 \times 2$, which resulted in a plate scale of $0.332 /$ pix.

2. The Complejo Astronómico El Leoncito (CASLEO): In the night January 25, 1996 CCD $U B V$ observations of NGC 2588 were performed using the $215 \mathrm{~cm}$ telescope. The typical seeing was about $2^{\prime \prime}$. The CASLEO camera uses a Tek-1024 detector that together with a focal reducer covers a round area with a 9.0 diameter and yields a plate scale of $0{ }^{\prime} 813 /$ pix.

3. The Cerro Tololo Inter-American Observatory (CTIO): $U B V(R I)_{C}$ CCD photometric data of the field of NGC 2588 were acquired with the $0.9 \mathrm{~m}$ telescope on the night of January 1, 1998.

Details of the observed fields and exposure times of the CASLEO and ESO observations (including standard star frames) are listed in Table 2 together with the expressions and coefficients of the calibration equations. Details of the observations and the reductions for the CTIO data are extensively given in Moitinho (2001). Figure 1 shows the areas of NGC 2580 and NGC 2588 covered on each run.

\subsection{Reductions}

The ESO and CASLEO data were reduced with the IRAF ${ }^{1}$ packages CCDRED, DAOPHOT, and PHOTCAL using the point spread function (PSF) method (Stetson 1987). Calibration coefficients for ESO data were obtained through observations of Landolt (1992) fields PG 0918+029, PG 0942-029, SA 098-562 and SA 101-424. For CASLEO observations, we used the PG 0918+029 and Rubin 149 fields also from Landolt (1992). In all cases, care was taken to have a good colour coverage. Exposure times, calibration equations and coefficients are given in Table 2, where $U B V I$ is used to indicate standard star magnitudes, $u b v i$ for the instrumental ones and $X$ denotes the airmass. As for the extinction coefficients, typical values for ESO and CASLEO were adopted. All data sets obtained from

\footnotetext{
${ }^{1}$ IRAF is distributed by NOAO, which are operated by AURA under cooperative agreement with the NSF.
}
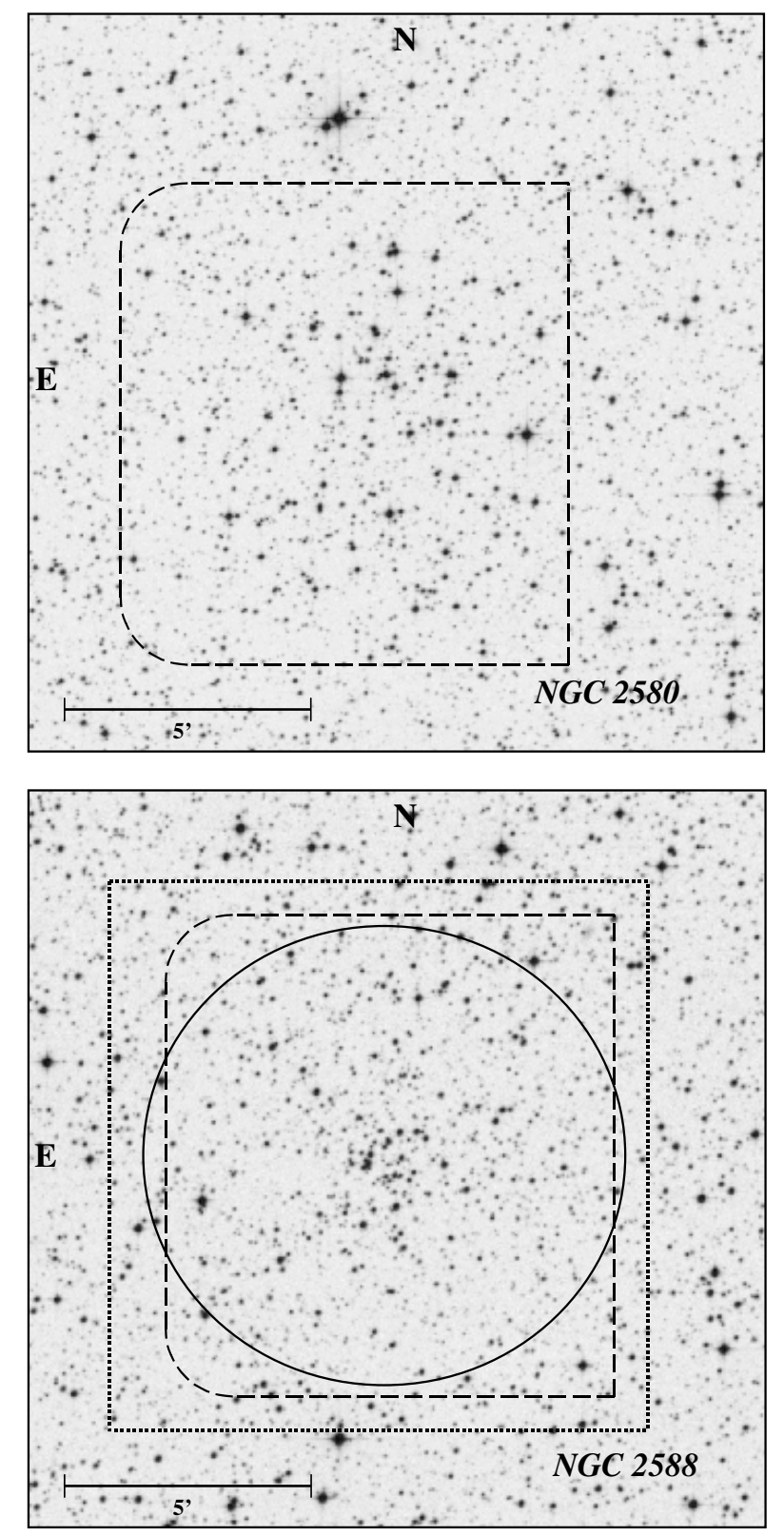

Fig. 1. Second generation Digitized Sky Survey (DSS-2), red filter images of the fields centred on NGC 2580 and NGC 2588 (see Table 1). Circles, dashed and dotted lines indicate areas covered by CASLEO, ESO and CTIO respectively.

different exposure times and different observational runs were put together using the DAOMASTER code (Stetson 1992). The photometric error trends against the $V$ magnitude of the combined data are shown in Fig. 2. The photometric data for some of the brightest stars of NGC 2580 and NGC 2588 are shown in Table 3. The full table is only available electronically at the CDS.

\section{Data analysis}

\subsection{Individual stellar coordinates}

Several of the brightest stars in the observed fields have precise astrometric measurements from the Tycho- 2 catalogue (Høg et al. 2000). Many other stars were also found in the 
Table 2. Exposure times used at ESO and CASLEO together with the adopted calibration equations and coefficients.

\begin{tabular}{|c|c|c|c|c|c|}
\hline \multicolumn{6}{|c|}{ Exposure times [s] } \\
\hline \multirow[b]{2}{*}{ Field } & \multicolumn{3}{|c|}{ CASLEO } & \multicolumn{2}{|c|}{ ESO } \\
\hline & $U$ & $B$ & $V$ & V & $I$ \\
\hline \multirow[t]{3}{*}{ NGC 2580} & - & - & - & 300 & 240 \\
\hline & - & - & - & 15 & 15 \\
\hline & - & - & - & 1 & 1 \\
\hline \multirow[t]{3}{*}{ NGC 2588} & 2700 & 1800 & 600 & 300 & 240 \\
\hline & - & 180 & 60 & 15 & 15 \\
\hline & - & 60 & 10 & 1 & 1 \\
\hline PG 0918+029 & 600 & 90 & 20 & 15 & 10 \\
\hline PG 0942-029 & - & - & - & 30 & 20 \\
\hline SA $098-562$ & - & - & - & 15 & 10 \\
\hline SA $101-424$ & - & - & - & 30 & 20 \\
\hline Rubin 149 & 600 & 30 & 10 & - & - \\
\hline \multicolumn{6}{|c|}{ Calibration equations } \\
\hline \multirow[t]{3}{*}{ CASLEO } & \multicolumn{5}{|c|}{$u=U+u_{1}+u_{2}(U-B)+u_{3} X$} \\
\hline & \multicolumn{5}{|c|}{$b=B+b_{1}+b_{2}(B-V)+b_{3} X$} \\
\hline & \multicolumn{5}{|c|}{$v=V+v_{1 b v}+v_{2 b v}(B-V)+v_{3 b v} X$} \\
\hline ESO & \multicolumn{5}{|c|}{$v=V+v_{1 v i}+v_{2 v i}(V-I)+v_{3 v i} X$} \\
\hline & \multicolumn{5}{|c|}{$i=I+i_{1}+i_{2}(V-I)+i_{3} X$} \\
\hline \multicolumn{6}{|c|}{ Calibration coefficients } \\
\hline \multicolumn{3}{|c|}{ CASLEO (25/01/1996) } & \multicolumn{3}{|c|}{ ESO } \\
\hline \multicolumn{3}{|c|}{$u_{1}=+7.595 \pm 0.025$} & \multicolumn{3}{|c|}{$v_{1 v i}=-0.560 \pm 0.023$} \\
\hline \multicolumn{3}{|c|}{$u_{2}=-0.230 \pm 0.041$} & \multicolumn{3}{|c|}{$v_{2 v i}=-0.058 \pm 0.023$} \\
\hline \multicolumn{3}{|l|}{$u_{3}=+0.49$} & \multicolumn{3}{|c|}{$v_{3 v i}=+0.135$} \\
\hline \multicolumn{3}{|c|}{$b_{1}=+2.611 \pm 0.006$} & \multicolumn{3}{|c|}{$i_{1}=-0.258 \pm 0.066$} \\
\hline \multicolumn{3}{|c|}{$b_{2}=-0.135 \pm 0.010$} & \multicolumn{3}{|c|}{$i_{2}=-0.063 \pm 0.070$} \\
\hline \multicolumn{3}{|c|}{$b_{3}=+0.27$} & \multicolumn{3}{|c|}{$i_{3}=+0.048$} \\
\hline \multicolumn{6}{|c|}{$v_{1 b v}=+1.406 \pm 0.004$} \\
\hline \multicolumn{6}{|c|}{$v_{2 b v}=+0.054 \pm 0.006$} \\
\hline \multicolumn{6}{|l|}{$v_{3 b v}=+0.12$} \\
\hline
\end{tabular}

2MASS catalogue. This information was used to compute equatorial coordinates for all the stars in each cluster field by means of a linear transformation. The coordinates are included in Table 3. The residuals of the transformation were of the order of $\sim 0.5$.

\subsection{Cluster angular radius}

Deriving the radial stellar density profile for a cluster requires the knowledge of the cluster centre. In our analysis we adopted the cluster centres given by Dias et al. (2002, see Table 1) which were confirmed by us, with an uncertainty of $\sim 0$ ' 2 , computing the position of the maximum of the marginal stellar distributions in both right ascension and declination over each cluster.

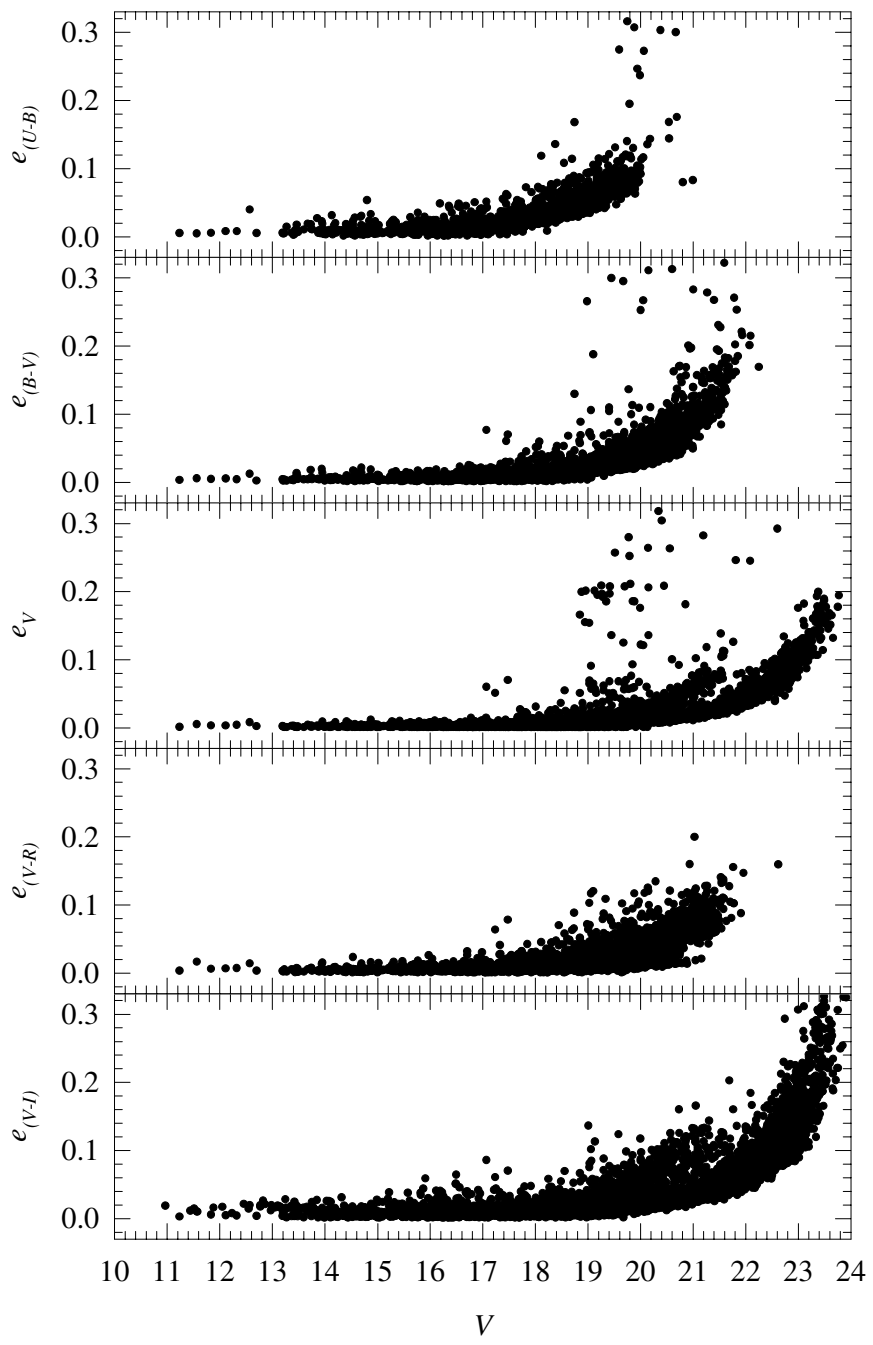

Fig. 2. DAOMASTER errors in the colour indexes and $V$ magnitude as a function of $V$.

The stellar density across each cluster was obtained using a) our CCD data which extend down to $V=20$, b) the corresponding DSS-2 red images of $20^{\prime} \times 20^{\prime}$ centred on each cluster (we estimate a $V_{\text {lim }} \sim 17-18$ ), and c) the 2 MASS infrared data $10^{\prime}$ around each cluster centre.

Stellar densities were obtained by counting the numbers of stars in a series of concentric rings 0.5 wide divided by their respective areas. Because of the limited area covered by our data some rings are not complete, so we adopted the corresponding densities as representative of the complete ring. Results are shown in Fig. 4. Analysis of the radial density profiles and determination of the clusters' radii will be discussed in Sect. 4.

\subsection{Cluster membership}

The most reliable assignment of cluster membership is achieved through the analysis of proper motions and radial velocities. For the fields of NGC 2580 and NGC 2588 most of these data are only available for foreground stars. Although there are some kinematical data for a few cluster stars, they have huge errors which render them useless in a membership 
Table 3. Some of the brightest stars of NGC 2580 and NGC 2588.

\begin{tabular}{|c|c|c|c|c|c|c|c|c|c|c|}
\hline \multicolumn{11}{|c|}{ NGC 2580} \\
\hline$\#$ & $\left.X{ }^{\prime \prime}\right]$ & $\left.Y{ }^{\prime \prime}\right]$ & $\alpha_{2000}$ & $\delta_{2000}$ & $V$ & $V-I$ & \multicolumn{2}{|c|}{ Remarks } & & \\
\hline 3 & -1.0 & 112.0 & $8: 21: 28.0$ & $-30: 16: 07.9$ & 11.44 & 0.45 & $\operatorname{lm}$ & \multicolumn{3}{|c|}{ TYC 71223851} \\
\hline 5 & -186.5 & 79.4 & 8:21:42.4 & $-30: 16: 40.6$ & 11.57 & 0.38 & $\operatorname{lm}$ & \multicolumn{3}{|c|}{ TYC 7122871} \\
\hline 6 & -4.2 & 161.2 & $8: 21: 28.3$ & $-30: 15: 18.7$ & 11.88 & 0.43 & $\operatorname{lm}$ & \multicolumn{3}{|c|}{ J08212833-301518 } \\
\hline 7 & -2.4 & -3.6 & $8: 21: 28.1$ & $-30: 18: 03.6$ & 12.05 & 0.30 & $\operatorname{lm}$ & \multicolumn{3}{|c|}{ TYC 712211291} \\
\hline 11 & 44.8 & 154.5 & $8: 21: 24.5$ & $-30: 15: 25.4$ & 12.55 & 0.30 & $\operatorname{lm}$ & \multicolumn{3}{|c|}{ J08212455-301525 } \\
\hline 35 & -51.3 & -200.8 & $8: 21: 31.9$ & $-30: 21: 20.7$ & 13.59 & 0.25 & $\operatorname{lm}$ & \multicolumn{3}{|c|}{ J08213194-302120 } \\
\hline 44 & -132.9 & 56.1 & $8: 21: 38.2$ & $-30: 17: 03.9$ & 13.84 & 0.31 & $\operatorname{lm}$ & \multicolumn{3}{|c|}{ J08213825-301703 } \\
\hline 46 & -73.0 & 56.5 & $8: 21: 33.6$ & $-30: 17: 03.5$ & 13.86 & 0.39 & $\operatorname{lm}$ & \multicolumn{3}{|c|}{ J08213362-301703 } \\
\hline 61 & -37.3 & 27.5 & $8: 21: 30.8$ & $-30: 17: 32.5$ & 14.27 & 0.43 & $\operatorname{lm}$ & \multicolumn{3}{|c|}{ J08213086-301732 } \\
\hline 68 & -30.9 & -75.1 & $8: 21: 30.3$ & $-30: 19: 15.0$ & 14.41 & 0.36 & $\operatorname{lm}$ & \multicolumn{3}{|c|}{ J08213037-301914 } \\
\hline 69 & 61.3 & -12.7 & $8: 21: 23.2$ & $-30: 18: 12.7$ & 14.43 & 0.36 & $\operatorname{lm}$ & \multicolumn{3}{|c|}{ J08212326-301812 } \\
\hline 75 & -20.6 & 10.2 & $8: 21: 29.5$ & $-30: 17: 49.7$ & 14.65 & 0.36 & $\operatorname{lm}$ & \multicolumn{3}{|c|}{ J08212958-301749 } \\
\hline 84 & -12.3 & 30.5 & $8: 21: 28.9$ & $-30: 17: 29.4$ & 14.80 & 0.36 & $\operatorname{lm}$ & & & \\
\hline 86 & 33.2 & -50.4 & $8: 21: 25.4$ & $-30: 18: 50.3$ & 14.81 & 0.43 & $\operatorname{lm}$ & \multicolumn{3}{|c|}{ J08212544-301850 } \\
\hline 89 & 72.1 & -44.3 & $8: 21: 22.4$ & $-30: 18: 44.2$ & 14.86 & 0.36 & $\operatorname{lm}$ & \multicolumn{3}{|c|}{ J08212242-301844 } \\
\hline 91 & -128.4 & -22.0 & $8: 21: 37.9$ & $-30: 18: 21.9$ & 14.87 & 0.43 & $\operatorname{lm}$ & \multicolumn{3}{|c|}{ J08213786-301821 } \\
\hline 101 & -45.8 & 26.2 & $8: 21: 31.5$ & $-30: 17: 33.7$ & 14.98 & 0.44 & $\operatorname{lm}$ & \multicolumn{3}{|c|}{ J08213151-301733 } \\
\hline \multicolumn{11}{|c|}{ NGC 2588} \\
\hline \# & $X\left[{ }^{\prime \prime}\right]$ & $Y\left[{ }^{\prime \prime}\right]$ & $\alpha_{2000}$ & $\delta_{2000}$ & $V$ & $U-B$ & $B-V$ & $V-R$ & $V-I$ & Remarks \\
\hline 26 & 1.3 & 33.3 & $8: 23: 09.8$ & $-32: 57: 56.6$ & 13.89 & 1.10 & 1.41 & 0.76 & 1.51 & pm (red) \\
\hline 31 & 33.4 & 26.3 & $8: 23: 07.3$ & $-32: 58: 03.7$ & 13.97 & 0.33 & 0.36 & 0.20 & 0.46 & $\operatorname{lm}$ \\
\hline 38 & 4.0 & 2.2 & 8:23:09.6 & $-32: 58: 27.8$ & 14.11 & 0.29 & 0.33 & 0.18 & 0.43 & $\operatorname{lm}$ \\
\hline 41 & -5.2 & 143.7 & 8:23:10.4 & $-32: 56: 06.3$ & 14.15 & 0.20 & 0.38 & 0.21 & 0.48 & $\mathrm{pm}$ \\
\hline 44 & 126.8 & 61.6 & $8: 22: 59.9$ & $-32: 57: 28.4$ & 14.22 & 0.87 & 1.26 & 0.71 & 1.40 & pm (red) \\
\hline 47 & 21.9 & 34.1 & 8:23:08.2 & $-32: 57: 55.9$ & 14.35 & 1.16 & 1.36 & 0.72 & 1.41 & pm (red) \\
\hline 48 & -12.0 & 21.6 & 8:23:10.9 & $-32: 58: 08.4$ & 14.35 & 0.24 & 0.32 & 0.17 & 0.42 & $\operatorname{lm}$ \\
\hline 53 & -36.7 & -6.2 & $8: 23: 12.9$ & $-32: 58: 36.2$ & 14.43 & 0.27 & 0.32 & 0.18 & 0.44 & $\operatorname{lm}$ \\
\hline 56 & -5.2 & -19.5 & $8: 23: 10.4$ & $-32: 58: 49.4$ & 14.51 & 0.17 & 0.31 & 0.17 & 0.43 & $\operatorname{lm}$ \\
\hline 64 & -30.4 & -61.9 & $8: 23: 12.4$ & $-32: 59: 31.9$ & 14.61 & 0.22 & 0.40 & 0.22 & 0.49 & pm (bin?) \\
\hline 65 & 3.3 & -90.2 & 8:23:09.7 & $-33: 00: 00.1$ & 14.66 & 0.22 & 0.31 & 0.14 & 0.38 & $\operatorname{lm}$ \\
\hline 69 & 51.3 & 79.2 & 8:23:05.9 & $-32: 57: 10.8$ & 14.67 & 0.22 & 0.31 & 0.18 & 0.44 & $\operatorname{lm}$ \\
\hline 72 & -42.3 & -9.9 & $8: 23: 13.3$ & $-32: 58: 39.8$ & 14.69 & 0.89 & 1.24 & 0.69 & 1.40 & pm (red) \\
\hline 78 & 18.8 & -15.6 & $8: 23: 08.5$ & $-32: 58: 45.6$ & 14.76 & 0.28 & 0.32 & 0.18 & 0.43 & $\operatorname{lm}$ \\
\hline 80 & -3.2 & -97.7 & $8: 23: 10.2$ & $-33: 00: 07.7$ & 14.77 & 0.33 & 0.38 & 0.22 & 0.48 & pm (bin?) \\
\hline 83 & -57.2 & -5.8 & $8: 23: 14.5$ & $-32: 58: 35.8$ & 14.81 & 0.28 & 0.34 & 0.19 & 0.46 & $\operatorname{lm}$ \\
\hline 92 & -17.4 & 15.2 & $8: 23: 11.3$ & $-32: 58: 14.8$ & 14.95 & 0.25 & 0.32 & 0.17 & 0.43 & $\operatorname{lm}$ \\
\hline 96 & 114.8 & -17.6 & 8:23:00.8 & $-32: 58: 47.5$ & 14.99 & 0.26 & 0.35 & 0.20 & 0.48 & $\operatorname{lm}$ \\
\hline
\end{tabular}

Note: Table 3 that includes all the photometric measurements is available in an electronic version at the CDS.

analysis. Therefore, to assign membership, we used the classical photometric criterion (e.g., Baume et al. 2003; Carraro 2002) by a detailed comparison of the stellar positions in all the photometric diagrams.

The colour-magnitude diagram (CMD) of NGC 2580 is shown in Fig. 5. The colour-colour diagrams (CCDs) and CMDs for NGC 2588 are shown in Figs. 6 and 7, respectively. In Fig. 8 we present the 2MASS data CMDs for both clusters. The CCDs of Fig. 6 and the CMDs of Figs. 5a, 7a, $7 \mathrm{~b}, 7 \mathrm{~d}, 7 \mathrm{e}, 8 \mathrm{a}$ and $8 \mathrm{~d}$ include all the stars inside the adopted radius for the corresponding cluster (see Sect. 4). The diagrams shown in Figs. 5b, 7c, 7e, 8b and 8e were plotted using only stars placed around each cluster in the regions adopted as comparison fields and denoted "comparison field 1" (dotted areas in the finding charts of Fig. 3). The 2MASS CMDs in Figs. 8c and 8f show alternative comparison fields ("comparison field 2", see Sect. 5.2). These latter fields were chosen in such way that they cover equal sky surfaces as the corresponding clusters.

The CMDs show different $V_{\text {lim }}$ (from $\sim 19.5$ in $U-B$ to $\sim 22.5$ in $V-I$ ) reflecting the different observational setups used in the construction of our data set (see Sect. 2.1). The deep $V-I$ data came from the NTT.

Both clusters exhibit a clear, blue, main sequence (MS) above $V \approx 16-17$ and in the case of NGC 2588 there are some bright red stars that could be evolved members (see Sect. 4 for 

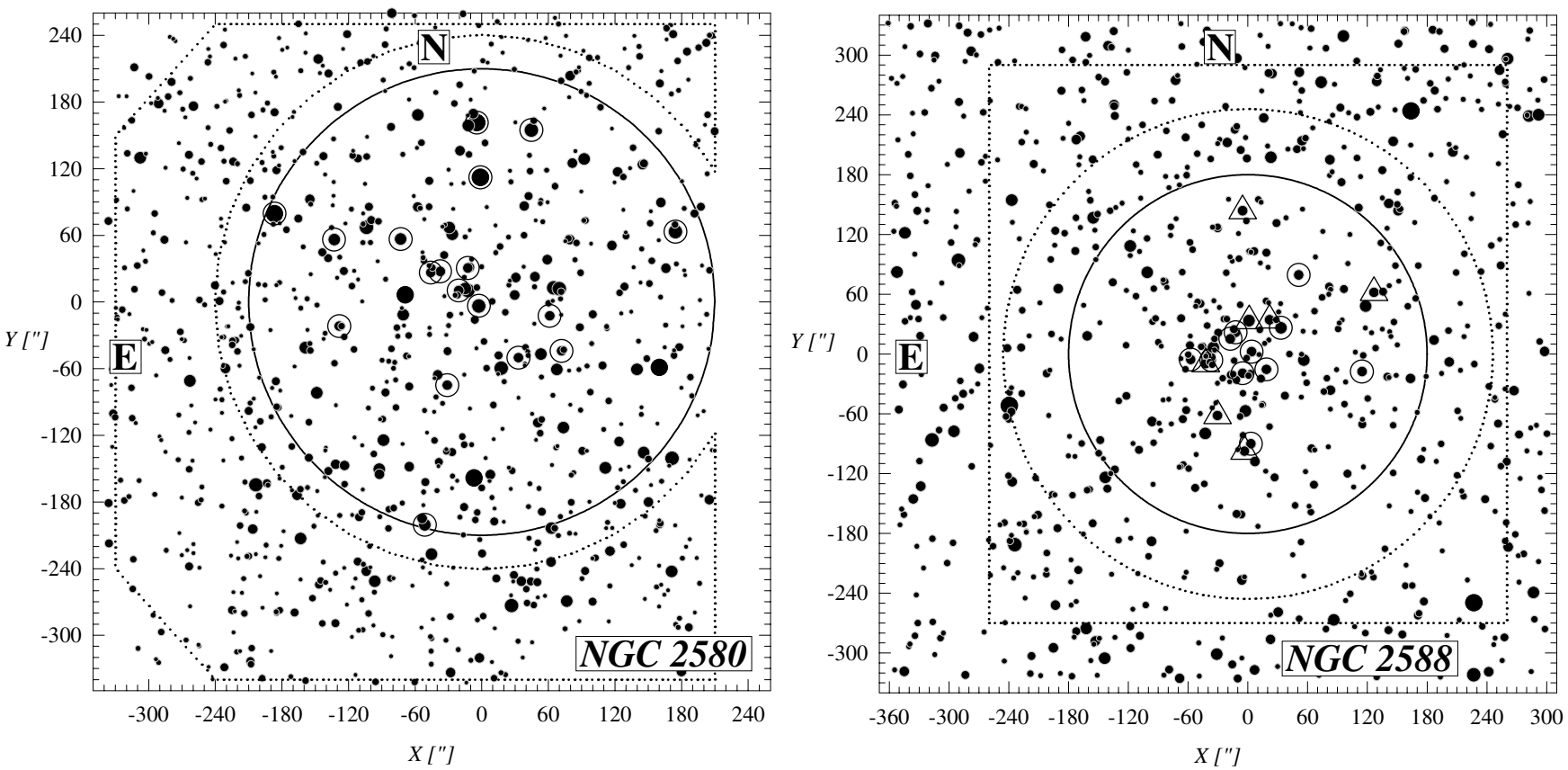

Fig. 3. Finding charts of the observed regions in NGC 2580 and NGC 2588 ( $V$ filter). The black solid circles, 3.5 in radius for NGC 2580 and 3.0 for NGC 2588 indicate the adopted angular sizes for the clusters (see Sect. 4 and Fig. 4). The dotted lines indicate the areas adopted as comparison fields. Adopted likely and probable cluster members with $V<15$ are indicated inside hollow circles and triangles, respectively. For a coordinate reference, the centre $(X=0 ; Y=0)$ corresponds to the cluster coordinates (see Table 1) and all $X-Y$ are expressed in arcseconds.

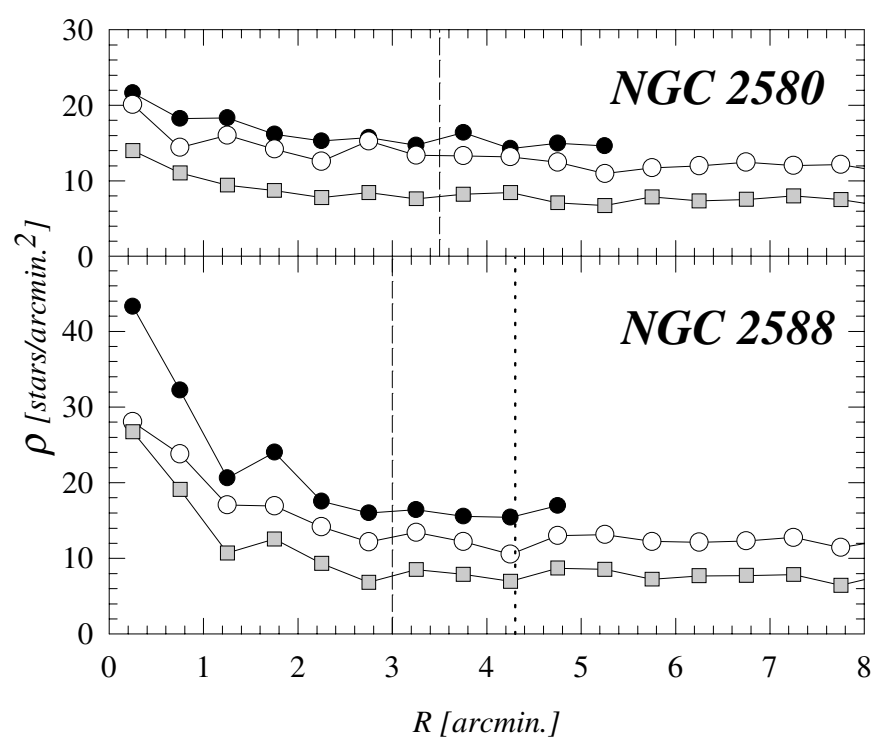

Fig. 4. Stellar surface density profiles in the regions of NGC 2580 and NGC 2588 as a function of radius. The plots represent our own data (black circles), DSS-2 images (white circles) and data from the 2MASS catalogue (grey squares). Dashed lines indicate the adopted limits for the clusters. The dotted line is probably a better limit for NGC 2588 (see Sect. 5.3).

details). This way, the individual positions for each star brighter than $V \sim 17$ are examined in all the photometric diagrams in the following way:

- for stars brighter than $V \sim 16$, if they have coherent locations near the ZAMS and/or adopted isochrones (see Sect. 3.4) they are adoped as likely members (lm);
- dimmer stars with magnitudes in the range $V \sim 16-17$ in the same conditions are considered only as probable members (pm);

- if some stars are brighter than $V \sim 16$, but off the MS, they are still considered as pm since their colour/magnitude offsets could be due to binarity or evolutionary effects (see Sects. 4.1 and 4.2);

- the number of stars in each magnitude bin is checked for agreement with the counts that are obtained from the sustraction between the "cluster fields" and the corresponding "comparison fields" (see Sect. 3.5).

At fainter magnitudes, contamination due to the Galactic field population (compare with CMDs of the "comparison fields") becomes severe, preventing us from easily identifying cluster members.

\subsection{Cluster parameters}

Following Moitinho (2001), it appears that the third Galactic quadrant is characterised by a reddening slope and a ratio of the total to selective absorption $\left(R=A_{\mathrm{V}} / E_{B-V}\right)$ that can be considered normal. Since the CCDs of NGC 2588 shown in Fig. 6 agree with the first assumption we adopted the $E_{U-B} / E_{B-V}=$ $0.72+0.05 E_{B-V}$ and $E_{V-I} / E_{B-V}=1.244$ (Dean et al. 1978) ratios and $R=3.1$ to shift the Schmidt-Kaler (1982) ZAMS over the CCDs and CMDs in order to obtain the corresponding colour excess and distance modulus for each cluster using the traditional ZAMS fitting procedure.

To estimate the clusters' ages, isochrones derived by Girardi et al. (2000) computed for solar metallicity, mass loss and overshooting were superposed onto the respective 


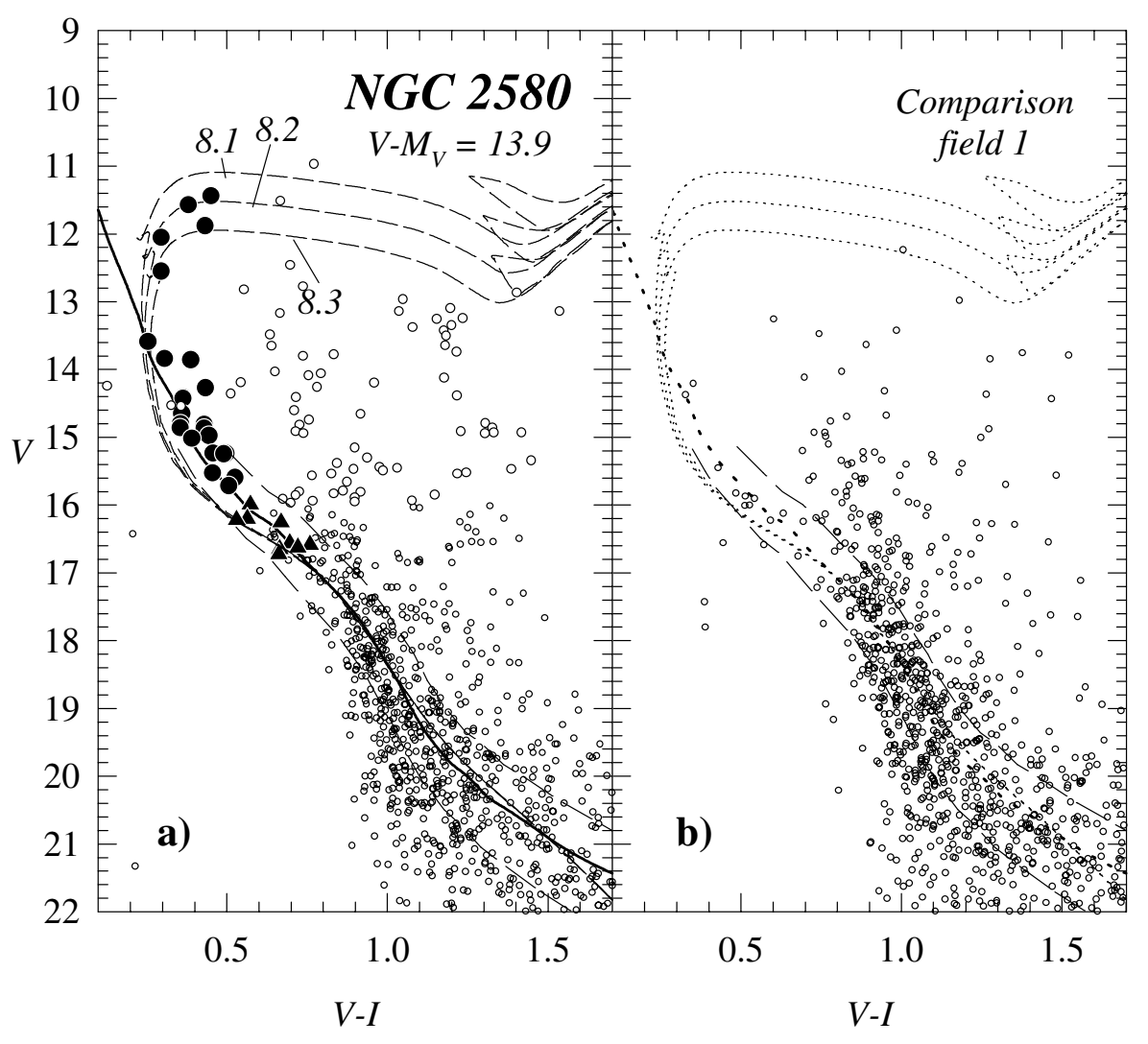

Fig. 5. Colour-magnitude diagrams (CMDs) of stars located inside the adopted radius of NGC 2580 and in the adopted comparison field. The symbols have the following meaning: black circles are adopted likely member stars ( $1 \mathrm{~m})$, black triangles are probable member stars (pm), white circles are non-member stars (nm), and small hollow circles are stars without any membership assignment. The solid line and dashed curves are the Schmidt-Kaler (1982) empirical ZAMS and isochrones from Girardi et al. (2002) respectively, corrected for the effects of reddening and distance. The adopted apparent distance modulus is $V-M_{V}=13.9\left(V-M_{V}=V_{0}-M_{V}+3.1 E_{B-V}\right.$, see Sect. 4). The numbers indicate $\log$ (age). Dotted lines showed on the comparison fields have the same meaning as the curves in the other panels. Long dashed lines in the $V$ vs. $V-I$ diagrams are the adopted envelopes used to compute the LF (see Sect. 3.5).

de-reddened CMDs. We looked for the ones producing the best fit over the stars placed along the MS and also over the possible red clump associated to each cluster. Also, from the location of adopted cluster member stars on the CCDs along the shifted ZAMS (dashed curves), we infer the spectral type corresponding to the earliest MS star and we use it as another indicator of the cluster ages.

\subsection{Cluster LF and IMF}

In this study, we present the Luminosity Function (LF) of each cluster - the distribution of stars over the luminosity range - in magnitude bins $1^{\mathrm{m}}$ wide. The IMF will be represented as the corresponding distribution of original ZAMS stellar masses in logarithmic bins.

To derive each LF, we first computed the apparent magnitude distribution of cluster stars and then shifted this distribution by using the derived cluster distance modulus. Since individual cluster memberships are only assigned for the brightest stars (see Sect. 4), our procedure is the following:

- for the brightest stars $(V<16)$ : we simply counted the number of likely member stars plus probable members on the red clump (if it does exist) and built the corresponding histogram of apparent magnitudes;
- for fainter stars $(V>16)$ : we adopted, as boundary limits, the envelopes around the MS on the $V$ vs. $V-I$ plane as shown in Figs. 5, 7e and 7f, and computed the apparent magnitude distribution of stars located in the cluster regions and also in the respective comparison fields. The last two distributions were subtracted from each other to obtain the brightness distribution of cluster stars.

Since the ESO observations are the deepest ones, we used them to compute the faint end of the LF. The completeness of these data was assessed by running several times the ADDSTAR task, adding $15 \%$ of the number of detected stars (following the same magnitude distribution), and by counting the number of stars recovered by the ALLSTAR task. In each run, a new set of artificial stars, following a similar magnitude distribution, was added at random positions. A comparison of the results from both tasks (taking into account that not all the stars are detected in both the $V$ and $I$ bands) yielded the completeness percentages shown in Table 4. These values are used to correct the apparent magnitude distributions of the corresponding fields and clusters. However we must stress that their significance is only important at very faint magnitudes $(V>21)$. 

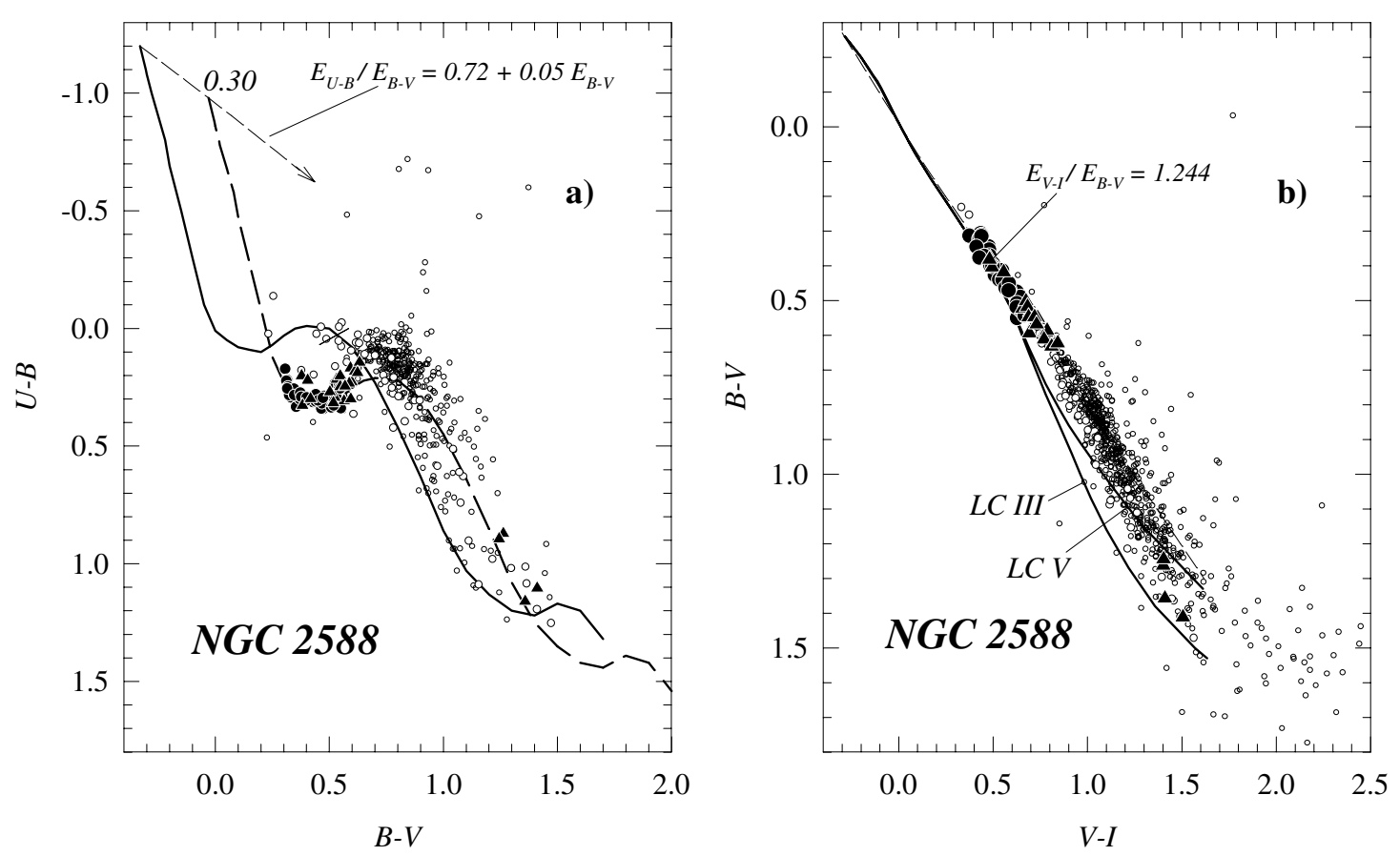

Fig. 6. Colour-colour diagrams (CCDs) of stars located inside the adopted radius of NGC 2588 a) $U-B$ vs. $B-V$ diagram. Symbols as in Fig. 5. The solid line is the Schmidt-Kaler (1982) ZAMS, whereas the dashed one is the same ZAMS, but shifted by the adopted colour excess (see Sect. 4). The dashed arrow indicates the normal reddening path. b) $B-V$ vs. $V-I$ diagram. Symbols and lines have the same meaning as in Fig. 5.

Table 4. Completeness analysis results for ESO data.

\begin{tabular}{crrr}
\hline \hline$\Delta V$ & NGC 2580 & \multicolumn{2}{c}{ NGC 2588 } \\
& & Cluster & \multicolumn{1}{c}{ Field } \\
\hline $15-16$ & $100.0 \%$ & $100.0 \%$ & $100.0 \%$ \\
$16-17$ & $99.8 \%$ & $99.8 \%$ & $99.8 \%$ \\
$17-18$ & $97.1 \%$ & $99.6 \%$ & $99.6 \%$ \\
$18-19$ & $96.7 \%$ & $98.4 \%$ & $99.1 \%$ \\
$19-20$ & $95.3 \%$ & $98.0 \%$ & $97.8 \%$ \\
$20-21$ & $93.6 \%$ & $95.9 \%$ & $96.8 \%$ \\
$21-22$ & $91.1 \%$ & $95.9 \%$ & $95.9 \%$ \\
$22-23$ & $73.2 \%$ & $85.9 \%$ & $87.0 \%$ \\
$23-24$ & $52.2 \%$ & $59.9 \%$ & $65.5 \%$ \\
\hline
\end{tabular}

As for the computation of the IMF, we considered two cases:

- stars placed along the MS; here we converted absolute magnitude intervals of the LFs into stellar masses using the mass-luminosity relation given by Scalo (1986);

- stars associated to the red clumps and those evolving off the MS; here we used the set of evolutionary tracks from Girardi et al. (2000) and traced back the individual path for each star to obtain its initial mass on the ZAMS (Baume et al. 1994).

The resulting apparent LFs and the IMFs are shown in Table 5 and Fig. 9 respectively.
Table 5. Apparent Luminosity Functions.

\begin{tabular}{ccc}
\hline \hline$\Delta V$ & NGC 2580 & NGC 2588 \\
\hline $12-13$ & 3 & - \\
$13-14$ & 2 & 2 \\
$14-15$ & 3 & 13 \\
$15-16$ & 9 & 10 \\
$16-17$ & 10 & 16 \\
$17-18$ & 7 & 18 \\
$18-19$ & 3 & 7 \\
$19-20$ & 13 & 11 \\
$20-21$ & 17 & -24 \\
$21-22$ & 20 & -40 \\
\hline
\end{tabular}

\section{Results}

\subsection{NGC 2580}

As NGC 2580 (=OCL $709=$ C0819-301) has been catalogued as an open cluster with a diameter of about $6^{\prime}$ (Dias et al. 2002), or 7' (Lyngå 1987), our CCD observations should completely cover this object (see Fig. 1) and part of its surroundings. van den Berg \& Hagen (1975) classified this cluster as "poor" (P) and more visible on the blue plates than on the red ones, whereas later on, Lyngå (1987) classified it as a Trumpler class II $2 \mathrm{~m}$ (slight concentration, medium range in brightness and medium richness), confirmed by Dias et al. (2002).

The radial stellar density profile shown in Fig. 4 reveals that we are dealing with a sparse object, in agreement with 

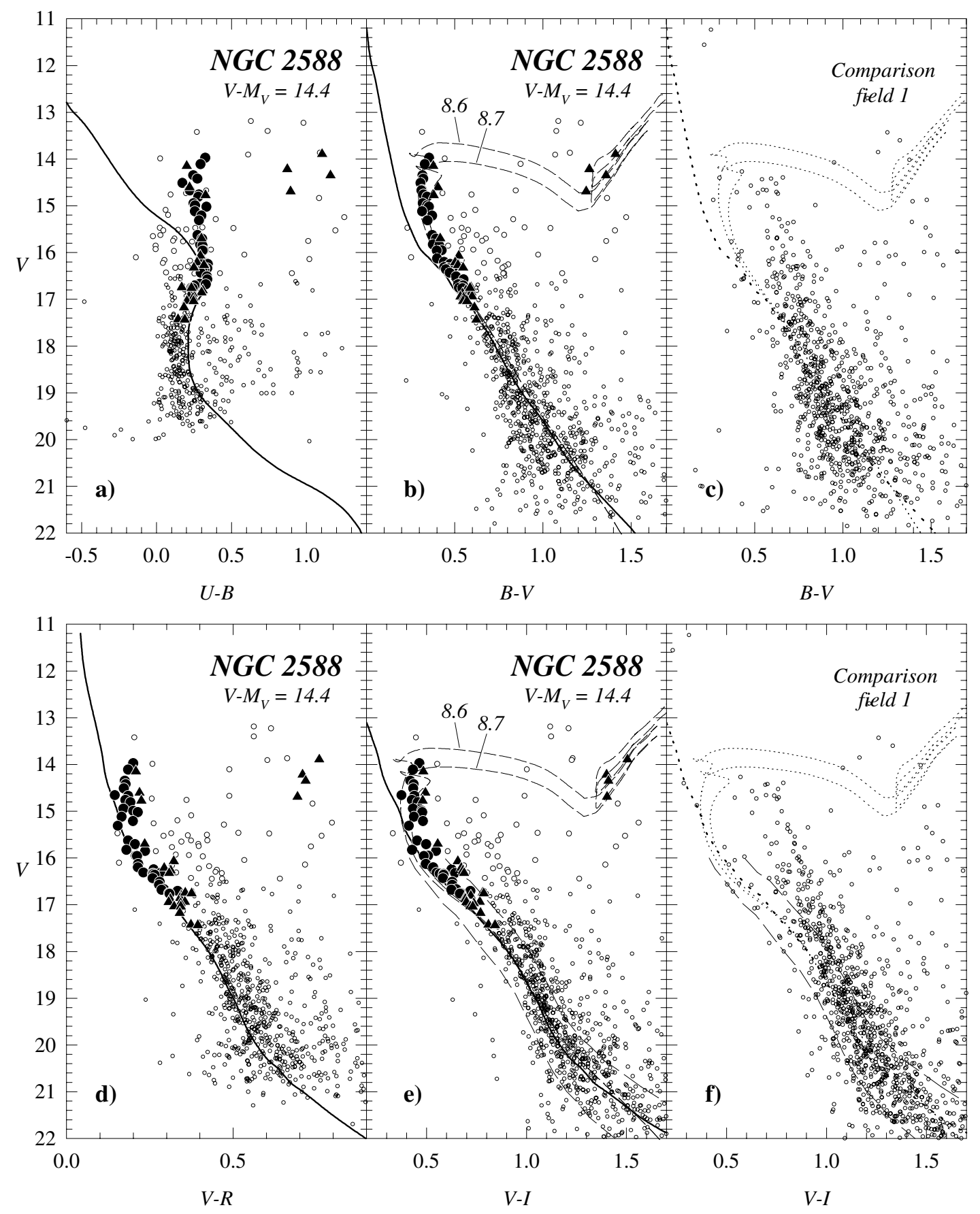

Fig. 7. CMDs for NGC 2588 and its comparison field. Symbols and lines have the same meaning as in Fig. 5.

its previous classification. According to this figure the density profile reaches the background field star density level at $\sim 3.5$. We adopted this value as the cluster angular diameter, in total agreement with the previous qualitative suggestion given by Lyngå (1987).

The CMD (Fig. 5) exhibits a blue sequence which we identify as the upper part of the MS of NGC 2580, extending from $V \approx 11$ to $V \approx 17$ and from $V-I \approx 0.25$ to $V-I \approx 0.7$. Among these stars, we adopt those with $V<16$ as likely members (lm) whereas the fainter ones are considered probable cluster members (pm). The resulting membership assignments are indicated with different symbols.
After some trials, the best fits of the Schmidt-Kaler (1982) ZAMS and the Girardi et al. (2000) isochrones to the stars adopted as $\mathrm{lm}$ and $\mathrm{pm}$ is obtained for a colour excess of $E_{V-I}=0.35 \pm 0.05\left(E_{B-V}=0.28 \pm 0.04\right)$, an apparent distance modulus $V-M_{V}=13.9 \pm 0.2$ (errors from eye inspection) and $\log ($ age $) \approx 8.2$. These values place NGC 2580 at $4.0 \pm 0.3 \mathrm{kpc}$ from the Sun with an age of about $160 \mathrm{Myr}$. According to Meynet et al. (1993), the brightest blue stars would be late Band early A- subgiants.

The apparent LF of NGC 2580 indicated in Table 5 presents two dips: the first at $\Delta V=13-14$ and the second at $\Delta V=$ $18-19$, confirming the visual impression given by the cluster's 

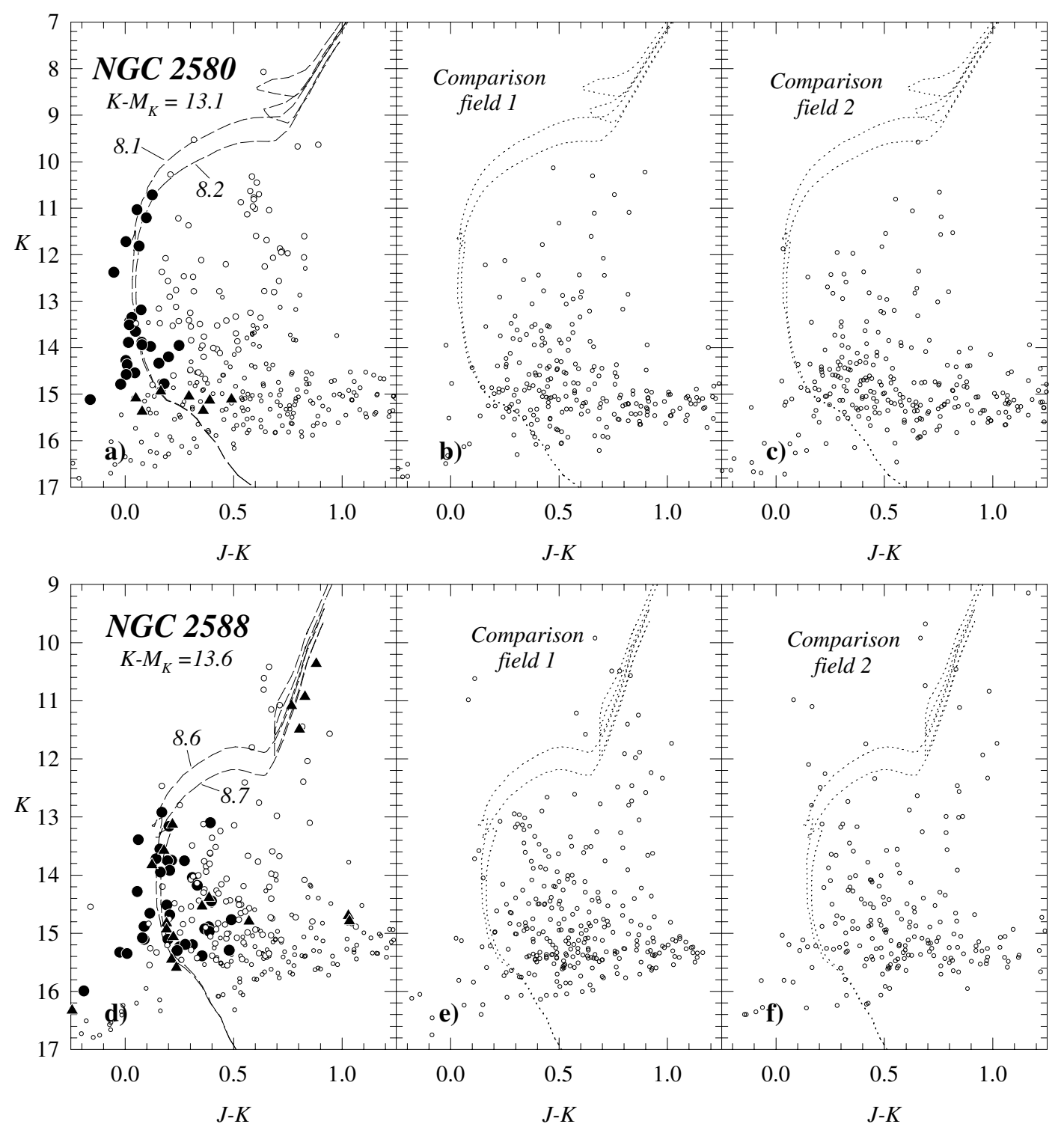

Fig. 8. $K$ vs. $J-K$ diagrams for stars inside the adopted radius for NGC 2580 and NGC 2588 and their comparison fields. Symbols are as in Fig. 5. Dashed curves are the isochrones from Girardi et al. (2002), corrected for reddening and for the adopted apparent distance moduli $\left(K-M_{K}=V_{0}-M_{V}+(3.1-2.8) E_{B-V}\right.$, see Sect. 4). The numbers indicate log(age). The dotted lines on the comparison fields have the same meaning.

CMD. In particular, the former is quite evident in both CMDs (Figs. 5 and 8a). The IMF of NGC 2580 is shown in the upper part of Fig. 9, and its slope, from a weighted least squares fit, is $x=1.50 \pm 0.48$ (solid line) for $7.5<M / M_{\odot}<1.5$ or $x=$ $1.31 \pm 0.45$ (dashed line) for the same range but excluding the bin related to the gap.

\subsection{NGC 2588}

NGC 2588 (=OCL $715=$ C0821-328) is a compact group of faint stars within a diameter of about 1.5 (Dias et al. 2002) or 2' (Lyngå 1987). So, at first view, our CCD observations should completely cover this object (see Fig. 1) and also a representative part of the field around it. According to van den Berg \& Hagen (1975), the richness of this cluster is "moderate" (M) and it is more noticeable on the blue plates than on the red ones. From the Lyngå (1987) classification, this object is a Trumpler class II $1 \mathrm{p}$ (slight concentration, most stars of nearly the same brightness and poor).

Figure 4 exhibits a well shaped radial stellar density profile for this cluster, with the highest star density inside the first $2^{\prime}$ from the centre and reaching the field star density level at $\sim 3^{\prime}$; this value is the one we adopted as the cluster radius. This way, the obtained diameter is larger than the one qualitatively suggested by Lyngå (1987) and Dias et al. (2002) that seem to only represent the core of this object.

Inspection of the different photometric diagrams (Figs. 6 and 7) allows us to identify the stars that compose a cluster sequence extending from $V \approx 14$ to $V \approx 17$ and from $B-V \approx 0.3$ to $B-V \approx 0.6$. As in the case of NGC 2580, we adopt those 


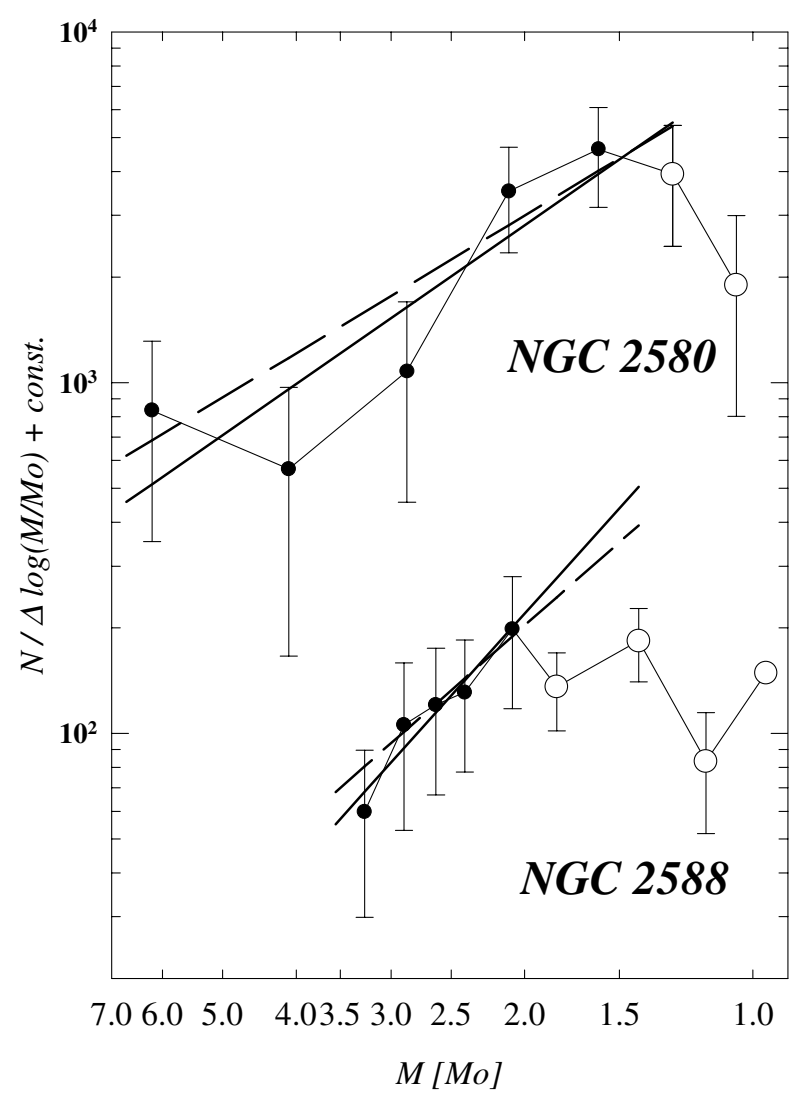

Fig. 9. Initial Mass Function (IMF) of NGC 2580 and NGC 2588. Error bars are from Poisson statistics. The weighted least square fittings for the more massive bins are indicated by solid and dashed right lines (open symbols indicate bins not used in the fits. See text for details). For display purposes the IMFs are shifted by an arbitrary constant.

with $V<16.5$ as likely members $(1 \mathrm{~m})$ whereas the fainter stars and those slightly above the sequence are considered probable cluster members (pm). Besides, the comparison to the best isochrones fits shown in Figs. $7 \mathrm{~b}$ and $7 \mathrm{e}$ allows the identification of some bright red stars (without a counterpart in the corresponding CMDs of the comparison field, Figs. 7c and 7f) that constitute the red clump associated to this cluster. However, since this region of the CMDs is affected by a certain degree of contamination by field stars, we add these candidate red-clump stars to our list of probable cluster members (pm).

The colour excess and apparent distance modulus for this cluster, according to the best fit to the stars adopted as $1 \mathrm{~m}$ and pm (see Figs. 7a, 7b, 7d and 7e), are $E_{B-V}=0.3 \pm 0.02$ and $V-M_{V}=14.4 \pm 0.1$ (errors from eye-inspection). These results place NGC 2588 at $4.95 \pm 0.2 \mathrm{kpc}$.

Regarding the age of this cluster, comparison to the isochrones in Figs. 7b and 7e reveals that NGC 2588 is about $450 \pm 50$ Myr. Moreover, according to the CCDs, the earliest MS stars in NGC 2588 have spectral type A0, which agrees with the age of $450 \mathrm{Myr}$ given above (Meynet et al. 1993).

Similarly to NGC 2580, the apparent LF of NGC 2588, as indicated in Table 5, shows a structure with two dips: the first appears at $\Delta V=15-16$ and the second at $\Delta V=18-19$. The former reveals a gap in the MS that is apparent in all the CMDs of this cluster, but the latter can be due to over-subtraction of the comparison field which could also explain the negative star counts obtained at faint magnitudes (see the following section for details). Regarding the IMF (see Fig. 9), the slopes determined from a weighted least squares fit are: $x=2.41 \pm 0.37$ (solid line) for $2.0<M / M_{\odot}<3.5$, or $x=1.90 \pm 0.33$ (dashed line) for $2.0<M / M_{\odot}<3.0$.

\section{Discussion}

\subsection{General concepts}

NGC 2580 and NGC 2588 are two young/middle age open clusters located in the third Galactic quadrant. As previously mentioned, the determination of their fundamental parameters gives us valuable clues for better understanding the structure of this region of our Galaxy and its star formation history. Furthermore, it is possible to obtain useful information concerning to the dynamical evolution of the open clusters themselves whether internal or in relation to the Galactic disk environment.

\subsection{The Vela-Puppis region}

Despite not being very young, NGC 2580 and NGC 2588 can still be used as spiral arm tracers. Putting together the data for these two clusters with those taken from the literature (e.g. Moffat \& Fitzgerald 1974; Moffat \& Vogt 1975; Moitinho 2002) for other clusters placed in the same region provides a clearer picture of the structure of the Vela-Puppis region: NGC 2588 is located, together with Haffner 18 (Munari et al. 1998), Haffner 19 (Munari \& Carraro 1996), Ruprecht 32 (Moffat \& Vogt 1975), Ruprecht 44 (Moffat \& Fitzgerald 1974), NGC 2453 (Moitinho 2002) and Ruprecht 55 (Bosch et al. 2003), in a place of the Galaxy that can be considered an extension of the Perseus arm. As for NGC 2580, it is located near Haffner 16 (Moitinho 2002). These two clusters are located closer to the Sun than those mentioned above, in the inter-arm region beyond the third quadrant extension of the local arm.

\subsection{Cluster IMFs}

It is evident from Fig. 9 that the IMFs of NGC 2580 and NGC 2588 cannot be represented by a unique power law along their entire mass range (as in NGC 4815, Prisinzano et al. 2001). The slopes for both clusters, derived from the most massive bins, are given at the end of Sects. 4.1 and 4.2 respectively.

Several studies of different clusters suggest that the spread in IMF slopes for stellar masses above $\approx 1 M_{\odot}$ is quite large but we do not have a clear idea yet of its true meaning: a) the IMF is not universal or b) there are intrinsic mistakes in its computation (Scalo 1998). Despite that, the slope values assumed as normal are $x=1.35$ (Salpeter 1955) for field stars or $x=1.7$ (Scalo 1998) for $1<M / M_{\odot}<10$. In the case of the cluster NGC 2580 the slope of the IMF agrees with such values but 
for NGC 2588 it turns out to be quite large, though still comparable to the typical slope of open clusters in the two oldest "age groups" ( $x \approx 2.2-2.3)$ found by Tarrab (1982). Anyway, as Prisinzano et al. (2001) argue, in this kind of old objects, dynamical evolution plays a decisive role since there has been enough time to disturb the original cluster mass distribution. Furthermore, as discussed by several authors (e.g., Sagar \& Richtler 1991; Kroupa et al. 1992; Scalo 1998) the effect of unresolved binaries and mass segregation should flatten the derived IMF.

For $M<2 M_{\odot}(\mathrm{NGC} 2588)$ and $M<1.5 M_{\odot}(\mathrm{NGC} 2580)$ the slopes of the IMF appear inverted. Since this could be an artifact caused by an improper choice of the comparison fields, which might not be representative of the true stellar field across the cluster surfaces, we selected an additional comparison field for each cluster using data from the 2MASS. These new comparison fields are placed farther from the centre of each cluster ( 7'.5 for NGC 2580 and 5'.5 for NGC 2588) and are indicated as "comparison field 2" in Fig. 8.

The CMDs of "comparison field 1" and "2" for NGC 2580 (Figs. 8b and 8c) are very similar, but for NGC 2588 the CMD of the "comparison field l" (Fig. 8e) appears more populated than that of "comparison field 2" (Fig. 8f), especially notably in the region of the lower MS. This evidence supports the choice of the comparison field used for NGC 2580 as representative of the true cluster field contamination, and its IMF reveals that it is deficient in low mass stars. However, for NGC 2588, the rather evident differences in the composition of the two comparison fields (Figs. 8e and 8f) leads us to consider the negative values in the faintest bins of its LF (see Table 5) as produced by field over-subtraction. In turn, this over-subtraction indicates that the cluster has undergone significant mass segregation and that several of its members can be found beyond our first estimate of the cluster size $(R=3.0)$; in fact it could reach out to $R=4.2$ (see Fig. 4).

The observed mass segregation may be the result of dynamical evolution or an imprint of the star formation process or the combined effect of both. In order to check if dynamical evolution plays an important role, we compute the dynamical relaxation times $\left(T_{E}\right)$ given by the following expression (Spitzer \& Hart 1971):

$T_{E}=\frac{8.9 \times 10^{5} N^{1 / 2} R_{\mathrm{h}}^{3 / 2}}{\left\langle M_{*}\right\rangle^{1 / 2} \log (0.4 N)}$

where $R_{\mathrm{h}}$ is the radius containing half of the cluster mass, $N$ is the number of cluster members and $\left\langle M_{*}\right\rangle$ is the average mass of the cluster stars. Since $N$ and $\left\langle M_{*}\right\rangle$ are lower and upper limit approximations, respectively, the obtained $T_{E}$ is indeed a rough lower estimate. For NGC 2588 we adopted $R_{\mathrm{h}}=2.9 \mathrm{pc}$ $\left(4^{\prime} / 2\right.$ at $\left.4.95 \mathrm{kpc}\right), N=77$ and $\left\langle M_{*}\right\rangle=1.8 M_{\odot}$ obtaining $T_{E}=19.2$ Myr. Comparing this value with the isochrone cluster age we find Age/ $T_{E} \approx 25$, which confirms that NGC 2588 is dynamically relaxed and the mass segregation effect due to dynamical evolution must be important. A similar analysis for NGC 2580 with $R_{\mathrm{h}}=2.2 \mathrm{pc}\left(2^{\prime}\right.$ at $\left.3.8 \mathrm{kpc}\right), N=50$, $\left\langle M_{*}\right\rangle=1.9 M_{\odot}$ yields $T_{E}=11.6 \mathrm{Myr}$ and Age $/ T_{E} \approx 10$. Therefore NGC 2580 is not as affected by dynamical effects as NGC 2588.

\subsection{Gaps in the main sequences}

A peculiarity that appears in the LFs, and also in the CMDs, is the presence of gaps at $V \sim 13$ for NGC 2580 and $V \sim 15.5$ for NGC 2588. They correspond to $M_{V} \sim-1\left(M \sim 5.2 M_{\odot}\right)$ and $M_{V} \sim+1\left(M \sim 2.5 M_{\odot}\right)$ respectively. Although the gaps are narrower than those detected in other clusters such as NGC 3766 (Moitinho et al. 1997), NGC 2571 (Giorgi et al. 2002), Tr 1 or Be 11 (Yadav \& Sagar 2002), they seem to be significant. To check this, we compute the probability that a lack of stars in a mass interval is a result of random processes (see Scalo 1986; Giorgi et al. 2002 for details) by using the following expression:

$P_{\text {gap }}=\left(M_{\text {sup }} / M_{\text {inf }}\right)^{(-N x)}$

where $M_{\text {sup }}$ and $M_{\text {inf }}$ are the border masses of the gaps ( $M_{\text {sup }}>$ $\left.M_{\text {inf }}\right), x$ is the exponent of the IMF and $N$ the number of cluster members located above the gap (with $M>M_{\text {sup }}$ ). For NGC 2580 the adopted values are $M_{\text {sup }}=6.1$ (star \# 11), $M_{\text {inf }}=3.9$ (star \# 35), $N=5$ and $x=1.31$. In the case of NGC 2588 they are $M_{\text {sup }}=2.6$ (star \# 118), $M_{\text {inf }}=2.3$ (star \# 149), $x=1.9$ and $N=15$ or 19 (depending on whether the red clump stars are considered or not).

The resulting probabilities are 5\% for NGC 2580 and $3 \%$ $(N=15)$ or $1 \%(N=19)$ for NGC 2588 . The obtained values are low enough to suggest that the gaps in both cluster MSs may be real features.

\section{Conclusions}

We have presented the first detailed CCD study of the two poorly known southern open clusters NGC 2580 and NGC 2588. In brief, we have found that:

- NGC 2580 is a slightly spread-out and moderately bright open cluster with a radius of 3.5 and located approximately at $d=4 \mathrm{kpc}$. This cluster is then placed in the inter-arm region between the local arm and the extension of the Perseus arm. We estimated a cluster reddening of $E_{B-V}=0.28$ and an age of $160 \mathrm{Myr}$.

- NGC 2588 is more concentrated and fainter than NGC 2580. Our analysis indicates that the core has a radius of about $1.5-2^{\prime}$, but the actual cluster could extend out to about 4.2 , indicating the presence of a corona formed by fainter (less massive) members, probably due to dynamical evolution. NGC 2588 is affected by a reddening of $E_{B-V}=0.3$. It is situated at about $5 \mathrm{kpc}$ from the Sun, in the extension of the Perseus arm and is about $450 \mathrm{Myr}$ old. The CMDs and LF of this cluster exhibit a gap at $V \sim 15.5$. Some red bright stars in the field are likely red-clump members which deserve future spectroscopic investigation.

This article is partially based on the Second Generation Digitized Sky Survey that was produced at the Space Telescope Science Institute under US government grant NAG W-2166. This study has also made use of: a) the SIMBAD database, operated at CDS, Strasbourg, France, and b) the Two Micron All Sky Survey, which is a joint project of the University 
of Massachusetts and the Infrared Processing and Analysis Center funded by NASA and NSF.

Acknowledgements. The authors acknowledge John Willis for his kind introduction at ESO NTT and for useful suggestions during the associated reduction of these data. We also thank the CASLEO staff for the technical support. A.M. acknowledges FCT (Portugal; grant PRAXIS XXI - BPD/20193/99). The work of GB is supported by Padova University through a postdoctoral grant.

\section{References}

Baume, G., Paoli, S., Vázquez, R. A., \& Feinstein, A. 1994, RMxAA, 29,212

Baume, G., Vázquez, R. A., Carraro, G., \& Feinstein, A. 2003, A\&AS, 402,549

Bosch, G., Barbá, R., Morrell, N., et al. 2003, MNRAS, 341, 169

Bessel, M. S. 2000, PASP, 112, 961

Carraro, G. 2002, MNRAS, 331, 785

Cousins, A. W. J. 1978a, MNSSA, 37, 62

Cousins, A. W. J. 1978b, MNSSA, 37, 77

Dean, J. F., Warren, P. R., \& Cousins, A. W. J. 1978, MNRAS, 183, 569

Dias, W. S., Alessi, B. S., Moitinho, A., et al. 2002, A\&A, 389, 871

Giorgi, E. E., Vázquez, R. A., Baume, G., Seggewiss, W., \& Will, J.-M. 2002, A\&A, 381, 884

Girardi, L., Bressan, A., Bertelli, G., \& Chiosi, C. 2000, A\&AS, 141, 371

Høg, E., Fabricius, C., Makarov, V. V., et al. 2000, A\&A, 357, 367

Kroupa, P., \& Tout, C. A. 1992, MNRAS, 259, 223
Landolt, A. U. 1992, AJ, 104, 340

Lyngå G. 1987, Catalog of Open Star Cluster Data (Strasbourg: CDS)

Meynet, G., Mermilliod, J.-C, \& Maeder, A. 1993, A\&AS, 98, 477

Moffat, A. F. J., \& Fitzgerald, M. P. 1974, A\&A, 34, 291

Moffat, A. F. J., \& Vogt, N. 1975, A\&A, 20, 85

Moitinho, A., Alfaro, E. J., Yun, J. L., \& Phelps, R. L. 1997, AJ, 113, 1359

Moitinho, A. 2001, A\&A, 370, 436

Moitinho, A. 2002, in Modes of Star Formation and the Origin of Field Populations, ed. E. K. Grebel, \& W. Brandner (San Francisco: Astronomical Society of the Pacific), ASP Conf. Proc., 285, 256

Munari, U., \& Carraro, G. 1996, MNRAS, 283, 905

Munari, U., Carraro, G., \& Barbon, R. 1998, MNRAS, 297, 867

Neckel, Th., \& Klare, G. 1980, A\&AS, 42, 251

Prisinzano, L., Carraro, G., Piotto, G., et al. 2001, A\&A, 369, 851

Sagar, R., \& Richtler, T. 1991, A\&A, 250, 324

Scalo, J. 1986, Fund. Cos. Phys., 11, 1

Scalo, J. 1998, in The Stellar Initial Mass Function, 38th Herstmonceux Conference, ASP Conf. Ser., 142, 201

Schmidt-Kaler, Th. 1982, Landolt-Börnstein, Numerical data and Functional Relationships in Science and Technology, ed. K. Schaifers, \& H. H. Voigt (Berlin: Springer Verlag), New Ser., Group VI, 2(b), 14

Spitzer, L., \& Hart, M. H. 1971, ApJ, 164, 399

Stetson, P. B. 1987, PASP, 99, 191

Stetson, P. B. 1992, in Stellar Photometry-Current Techniques and Future Developments, ed. C. J. Bulter, \& I. Elliot (Cambridge: Cambridge University Press), IAU Coll., 136, 291

Tarrab, I. 1982, A\&A, 109, 285

van den Berg, S., \& Hagen, G. L. 1975, AJ, 80, 11

Yadav, R. K. S., \& Sagar, R. 2002, MNRAS, 337, 133 